\title{
Effect of Ginger on Hyperglycemia Induced by Streptozotocin in Pregnant Rats and Postnatal Neurodevelopment of their Offspring
}

\author{
Mehouel Raouia ${ }^{* *}$, Ferhati Habiba ${ }^{1}$, Tahraoui Abdelkrim ${ }^{1}$ \\ 1Badji Mokhtar University, Department of Biology, Laboratory of Neuro-endocrinology Applied, BP 12, 23000, Annaba, \\ Algeria.
}

\begin{abstract}
This study was performed to investigate the consequences of severe maternal hyperglycemia induced by streptozotocin in Wistar rats on postnatal development of offspring. Besides, the ability of a ginger extract to restore glycemic balance in dams and prevent the appearance of disorders in offspring. Diabetes was induced in dams before pregnancy by a single injection of streptozotocin at a dose of $50 \mathrm{mg}$ / kg. Ginger was orally administered from the fourteenth (14th) to the nineteenth (19th) day of pregnancy. Two tests before and after weaning were performed to monitor the offspring development. Our study clearly showed that treatment of diabetic pregnant rats with a ginger extract lowers blood glucose levels and help prevent disorders in offspring.
\end{abstract}

Keywords: Maternal diabetes, Postnatal neurodevelopment, Streptozotocin, Wistar rats, Ginger

\section{INTRODUCTION}

Natural products include a variety of chemical compounds that have been evolutionarily selected for their ability to improve the survival of an organism. ${ }^{1}$

Because of the diversity of their biological activities, they have been widely used in the field of human health as a dietary supplement in traditional medicine for thousands of years. ${ }^{2}$

Ginger (Zingiber officinale) is a plant frequently used for thousands of years as a culinary seasoning, in particular in Asian cuisine, and is a common medicinal

*Corresponding Author: Mehouel Raouia, e-mail: mehouel.raouia@gmail.com Mehouel Raouia ORCID Number: 0000-0001-5749-2091

Ferhati Habiba ORCID Number: 0000-0002-6051-421X

Tahraouia Abdelkrim ORCID Number: 0000-0002-4855-4112

(Received 09 July 2019, accepted 05 November 2019) 
agent in the traditional medicine systems of China, India and other Eastern cultures. Ginger has several beneficial pharmacological effects (hypoglycemic, insulinotropic, hypolipidemic) in laboratory animals ${ }^{3}$ and in humans. ${ }^{4}$

It has been demonstrated that ginger or its extracts possess certain pharmacological activities, including analgesic effects ${ }^{5}$. Herbal medicines are considered as a fundamental source of new compounds based on pharmacological active principle, where about $11 \%$ of basic drugs in the 21 st century are derived exclusively from plant origin ${ }^{6}$.

In our study, we investigate the effect of maternal diabetes induced by streptozotocin in Wistar rats on postnatal neuro-development of offspring. Moreover, the effect of a ginger extract on maternal hyperglycemia and its impact on the development of offspring.

\section{METHODOLOGY}

\section{Experimental Animals}

Wistar strain female and male (that will be used for mating) weighing $240 \pm 10$ g were obtained from Pasteur Institute (Algiers, Algeria). The rats were housed in clean polyethylene cages $(58 \times 38 \times 19 \mathrm{~cm}, 5$ rats per cage for each sex $)$ and maintained at standard facility conditions of temperature $\left(25 \pm 2{ }^{\circ} \mathrm{C}\right)$, humidity $(65 \pm 5 \%)$ with a photoperiod of $12 \mathrm{~h}$ light and $12 \mathrm{~h}$ dark cycle. The rats were fed with commercial chow and tap water ad libitum. Procedures for the care and handling of animals were in compliance with current international laws and policies (NIH Publication No. 85-23, 1985, revised 1996).

\section{Study desing}

After adaptation period, the female rats were divided into four (4) experimental groups, and treated as follows:

- Group 1: included control dams (C)

- Group 2: included dams treated with ginger extract (G)

- Group 3: included diabetic dams (D),

- Group 4: included diabetic dams treated with ginger extract (D + G)

\section{Animal Treatments}

\section{Diabetes Induction}

Streptozotocin (STZ) was obtained from Sigma - Aldrich chemicals (USA).

Diabetes was induced by a single intraperitoneal injection of a freshly prepared 
solution of STZ (5Omg/kg body weight) in $0.1 \mathrm{M}$ citrate buffer ( $\mathrm{pH} 4.5$ ). Animals were considered as diabetic if their blood glucose values were above $250 \mathrm{mg} /$ $\mathrm{dl}$ on the third day after STZ injection. The blood glucose levels were mesured from the rat tail vein ${ }^{7}$ by using Accucheck verio glucometer (United Kingdom).

\section{Ginger extract administration}

The ginger extract used in our experiment is a product of a leading Life Science and High Technology company (Sigma-Aldrich, United States)

(Ginger extract-naturel, Sigma-Aldrich, code: W 252108, CAS Number: 84696-15-1).

Ginger extract was conducted to pregnant rats in groups $(G)$ and (D + G) by oral gavage once daily in $80 \mathrm{mg} / \mathrm{kg}$ body weight/ day at $\mathrm{o} 8 \mathrm{~h}: 30 \mathrm{in} 6 \mathrm{consecu}-$ tive days, from the fourteenth (14th) to the nineteenth (19th) day of pregnancy.

\section{Mating}

The female rats of the different groups were individually housed in appropriate polyethylene cages and subjected to the first vaginal smears to determine estrus cycle phases based on a standard cytological analysis. ${ }^{8}$ Each pro-estrus female was placed overnight in cage with one male of the same strain. Second vaginal smears were examined the next morning and the presence of spermatozoa was considered as day 1 of pregnancy (gestationel day $1=$ GD 1 ).

\section{Farrowing}

Parturition day was designated as PND o. (PND = Postnatal day).

For the experimental study of offspring, we used 12 male and 12 female rats from each group.

\section{Postnatal development evaluation}

In order to monitor the postnatal development, the offspring were weighed in PND 1, PND7, PND 14 and PND 21.

\section{Tests applied in male and female pups (Before and after weaning) Negative geotaxis (PND8)}

The negative geotaxis test is supposed to evaluate motor coordination and the cerebellar integration. The test consists in placing the rattons at PND 8 on an inclined plane at an angle of 25 degrees to the horizontal and their heads pointed down the slope. The measured variable is the latency time for a rat to make a complete half-lap of 180 degrees, ending up at the top of the slope. (measurement of equilibration, maturation of the cerebellum and semicircular 
canals of the inner ear). ${ }^{9}$

\section{Object recognition test (PND 45)}

The test is performed in order to evaluate the recognition memory in rodents because it uses their natural preference for a new object compared to a familiar object. ${ }^{10}$

At postnatal 45, the offspring were placed in an open arena $(50 \times 50 \times 40)$ and allowed to freely explore two identical objects during a pretest of 5 minutes (exploration phase) and then returned to their cages. After a one-hour retention phase, the pups were resubmitted for 5 minutes at the test session (recognition phase) during which one of the two familiar objects previously explored was replaced by a new object. ${ }^{11}$ The recognition memory was evaluated during the test session and expressed by the recognition index which indicates the ratio of the exploration time of the new object on the exploration time of the two objects.

\section{Statistical analysis}

All results were expressed as the means \pm standard deviation $(\mathrm{M} \pm \mathrm{SD})$.

Statistical calculations were made using Minitab software (version 18.1.o.o, Minitab Inc., USA). The comparison between the different groups was performed using Student's t test.

\section{RESULTS}

\section{Maternal glycemia}

Table 1. Variation in maternal blood glucose during the experiment $(\mathrm{g} / \mathrm{l})$. Results are expressed as the mean \pm SD $(n=7)$.

\begin{tabular}{|c|c|c|c|c|c|c|c|}
\hline Groups & \multicolumn{2}{|c|}{ Before gestation } & \multicolumn{3}{c|}{ During gestation } & \multicolumn{2}{c|}{ After gestation } \\
\hline & $\begin{array}{c}\text { Before } \\
\text { induction }\end{array}$ & $\begin{array}{c}\text { After } \\
\text { induction }\end{array}$ & GD 1 & GD 14 & GD 19 & PND 1 & PND 21 \\
\hline C & $\mathbf{0 . 9 4 \pm 0 . 1 0}$ & $0.89 \pm 0.05$ & $0.91 \pm 0.04$ & $0.92 \pm 0.07$ & $0.90 \pm 0.11$ & $0.93 \pm 0.03$ & $0.88 \pm 0.12$ \\
\hline G & $\mathbf{0 . 9 0 \pm 0 . 1 1}$ & $0.92 \pm 0.07$ & $0.93 \pm 0.09$ & $0.94 \pm 0.04$ & $0.89 \pm 0.03$ & $0.90 \pm 0.08$ & $0.89 \pm 0.07$ \\
\hline D & $\mathbf{0 . 9 2 \pm 0 . 0 3}$ & $3.04 \pm 0.13$ & $3.20 \pm 0.12$ & $4.10 \pm 0.15$ & $4.32 \pm 0.17$ & $4.41 \pm 0.10$ & $4.71 \pm 0.23$ \\
\hline D+ G & $\mathbf{0 . 8 8} \pm \mathbf{0 . 0 5}$ & $3.08 \pm 0.09$ & $3.17 \pm 0.05$ & $4.13 \pm 0.12$ & $2.57 \pm 0.21$ & $2.53 \pm 0.14$ & $2.52 \pm 0.17$ \\
\hline
\end{tabular}


On the third day after the induction of diabetes, the blood glucose levels in STZ injected diabetic rats were significantly $(\mathrm{P}<0.001)$ increased than of normal control rats. However, we found that the elevated blood glucose levels in diabetic rats $(\mathrm{D}+\mathrm{G})$ were significantly $(\mathrm{P}<\mathrm{0.001})$ decreased after 6-days ginger administration compared to diabetic rats (D). (Table 1)

\section{Offspring body weight evolution}

Pups of diabetic dams (D) gained significantly less weight than those from control dams during the postnatal development $(p<0.001)$. However, pups of diabetic dams treated with ginger $(D+G)$ gained significantly more weight than those from diabetic dams during the postnatal development $(p<0.001)$. The difference at PND 21 is very highly significant ( $\mathrm{p}<0.001$ ), (Fig 1)

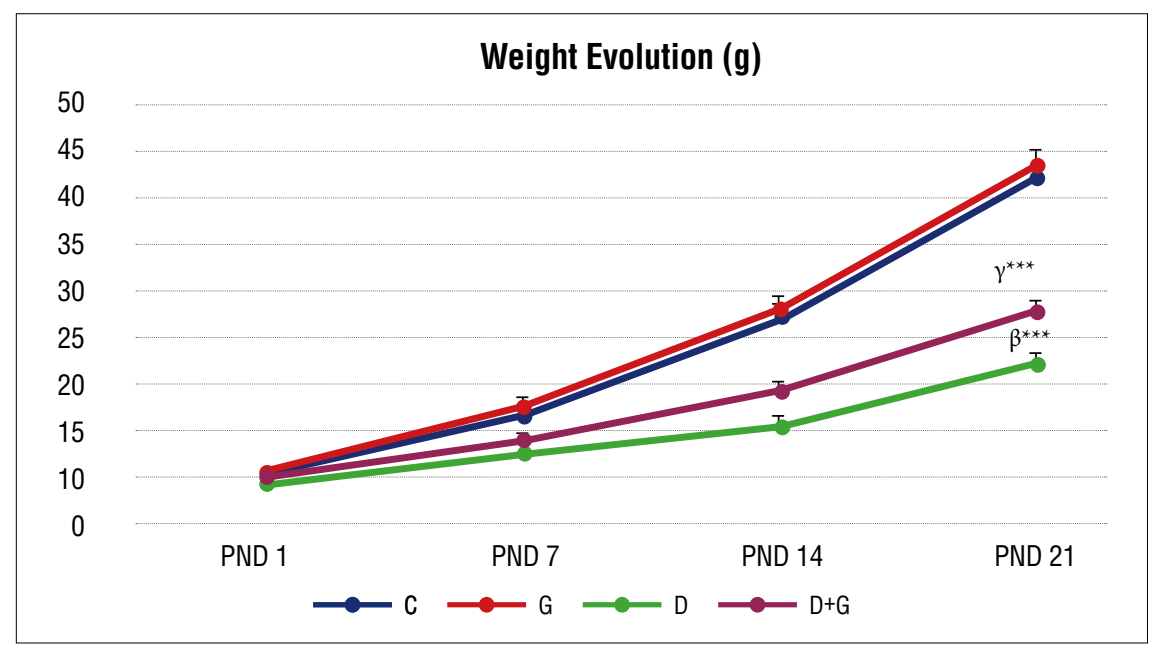

Figure 1. Offspring body weight change ( $\mathrm{g}$ ) during the development from PND 1 to 21. Results are expressed as the mean $\pm S D ; n=24$ (12 males, 12 females). ( $\beta$ : comparison vs C), ( $\gamma$ : comparison vs D).

\section{Body weight of male and female pups}

\begin{tabular}{|c|c|c|c|c|c|c|c|c|}
\hline \multirow{2}{*}{ Groups } & \multicolumn{2}{|c|}{ PND 1 } & \multicolumn{2}{c|}{ PND 7 } & \multicolumn{2}{c|}{ PND 14 } & \multicolumn{2}{c|}{ PND 21 } \\
\cline { 2 - 9 } & $\mathbf{M}$ & $\mathbf{F}$ & $\mathbf{M}$ & $\mathbf{F}$ & $\mathbf{M}$ & $\mathbf{F}$ & $\mathbf{M}$ & $\mathbf{F}$ \\
\hline C & $5.83 \pm 0.55$ & $5.66 \pm 0.62$ & $13.08 \pm 1.11$ & $12.66 \pm 1.02$ & $25.50 \pm 0.86$ & $24.08 \pm 1.32$ & $42.08 \pm 1.38$ & $40.58 \pm 1.18$ \\
\hline G & $5.94 \pm 0.44$ & $5.76 \pm 0.40$ & $14.25 \pm 0.59$ & $13.33 \pm 1.02$ & $26.75 \pm 0.72$ & $24.83 \pm 1.14$ & $44.41 \pm 0.49$ & $41.50 \pm 1.11$ \\
\hline D & $4.91 \pm 0.64$ & $5.16 \pm 0.37$ & $8.25 \pm 0.92$ & $8.75 \pm 1.09$ & $11.25 \pm 1.16$ & $11.91 \pm 1.03$ & $18.83 \pm 0.89$ & $19.66 \pm 0.94$ \\
\hline D + G & $5.33 \pm 0.47$ & $5.41 \pm 0.49$ & $9.75 \pm 0.59$ & $10.16 \pm 0.79$ & $15.08 \pm 0.64$ & $16.25 \pm 0.82$ & $24.50 \pm 0.86$ & $26.25 \pm 0.92$ \\
\hline
\end{tabular}

Table 2. Body weight of male and female offspring $(\mathrm{g})$. Results are expressed as the mean \pm $\mathrm{SD} ; \mathrm{n}=24$ (12 males, 12 females). 
Table 2 shows the body weight (g) of male and female offspring at day PND1, PND7, PND14 and PND21. We found a delay in weight growth in males of diabetic dams (D) compared to females. In PND 1 and PND 7 our results show no significant difference in body weight between males and females from diabetic (D) and diabetic ginger-treated dams (D + G) (P> 0.05). PND 14 and PND 21 showed a very highly significant increase in body weight of females from ginger-treated diabetic dams $(D+G)$ compared with males $(p<0.001)$. In PND 21, our results show a significant increase in body weight of females from diabetic dams (D) compared with males ( $\mathrm{P}<0.05$ ), (Table 2).

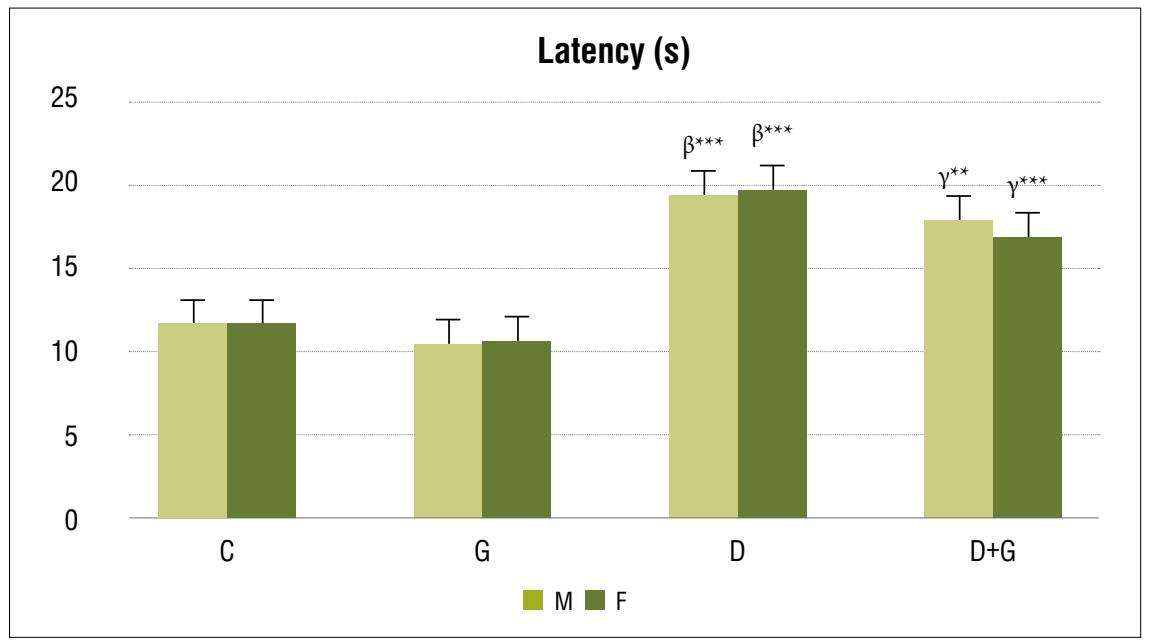

Figure 2. Latency time (s) in the negative geotaxis test at day PND 8. Results are expressed as the mean $\pm S D ; n=24$ (12 males, 12 females). ( $\beta$ : comparison vs $C),(\gamma$ : comparison vs $D)$

\section{Variation in postnatal neurodevelopment parameters of pups: Negative geotaxis}

According to this test, latency significantly increased ( $p<0.001)$ in male and female pups of diabetic dams (D) compared to pups of control dams. In contrast, highly significant decrease $(\mathrm{p}<0.01)$ in latency in male offspring of diabetic dams treated with ginger $(D+G)$ compared to male offspring from diabetic dams (D), while in female offspring, significant decrease $(\mathrm{p}<0.001)$. Latency significantly $(\mathrm{P}<0.05)$ decreased in female of diabetic dams treated with ginger $(\mathrm{D}+\mathrm{G})$ compared to males (Fig 2). 


\section{Object recognition test}

As depicted in Fig 3, recognition index significantly $(\mathrm{P}<0.001)$ decreased in males and females of diabetic dams as compared to pups of control dams. Significantly increase $(\mathrm{P}<0.001)$ of recognition index in offspring (male and female) of diabetic dams treated with ginger $(D+G)$ compared to offspring of diabetic dams (D), (Fig 3).

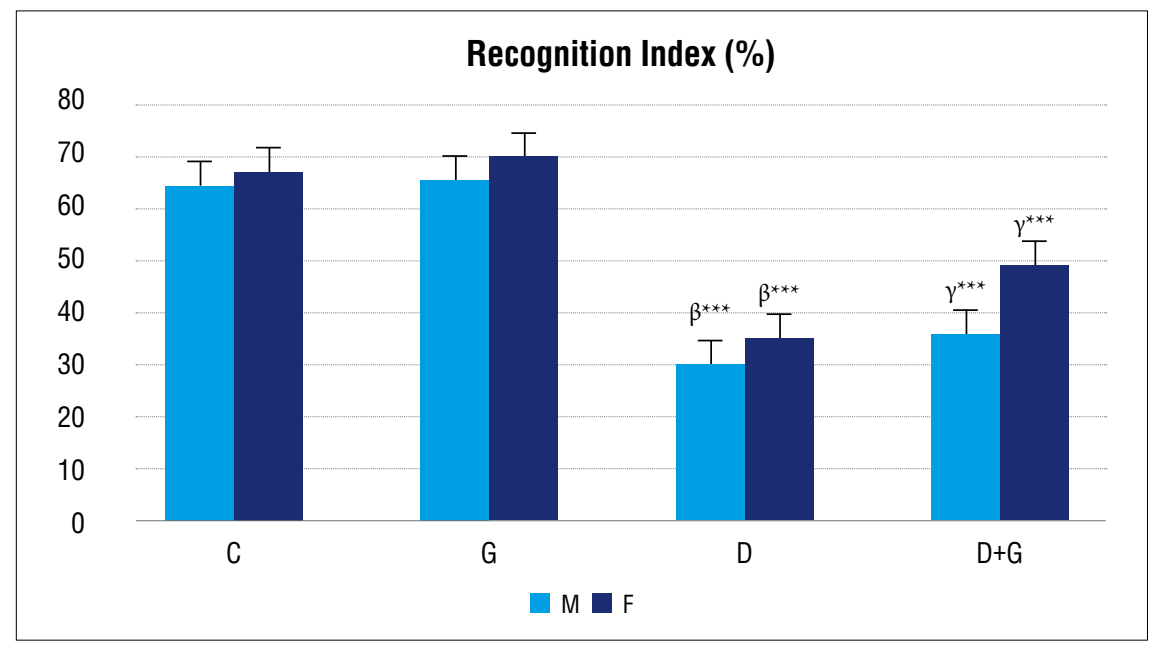

Figure 3. Recognition index in offspring at PND 45. Results are expressed as the mean $\pm S D$; $\mathrm{n}=24$ (12 males, 12 females). ( $\beta$ : comparison vs $C$ ), ( $\beta$ : comparison vs $D$ ).

Diabetes mellitus is a group of chronic metabolic disorder characterized by hyperglycemia that causes lesions affecting several devices or systems especially vessels and nerves. ${ }^{12}$

In this study we reported the effects of severe maternal hyperglycemia induced by streptozotocin on development of offspring and the ability of a ginger extract to restore glycemic balance in dams and prevent the onset of disorders in offspring.

Elevated maternal blood glucose in diabetic groups testifies the installation of the diabetic disease. ${ }^{13}$ According to the conclusions of several studies, this hyperglycemia is due to the effects of Streptozotocin by its action on pancreatic $\beta$ cells, leading to a reduction of the cell mass. ${ }^{14}$ Streptozotocin activates the expression of protein kinase $\mathrm{C}$, responsible for the dephosphorylation of the insulin receptor ${ }^{15}$

Ginger has been used traditionally to treat a large number of conditions such as fever and infectious diseases, abdominal pain, abdominal spasms, nausea and vomiting, motion sickness, arthritis, rheumatism, ulcerative colitis, gin- 
givitis hypertension and diabetes. ${ }^{16}$ The bioactive components of ginger were characterized by spectroscopic analysis in the form of zingerone, gingerdione, dehydrozingerones which had a potent antioxidant, shogaols, gingerols and a volatile oil. ${ }^{17}$

Despite the introduction of hypoglycaemic agents from natural and synthetic sources, diabetes and its complications remain a major medical problem in the world's population.

Currently, some medicinal plants are used to treat diabetes, and ginger is one of the most potent herbs traditionally used to treat diabetes mellitus. ${ }^{18}$

Treatment of diabetic pregnant rats $(D+G)$ with ginger extract for six consecutive days reduced glycemia. This is in agreement with previous reports. ${ }^{3}$

According to the literature, two models have been proposed to study diabetes in rodents during pregnancy and its implications for offspring. Moderate diabetes model causes mild hyperglycemia in mothers and macrosomia in the offspring, being similar to repercussions of diabetes type 2 and gestational diabetes mellitus. On the other hand, severe diabetes model causes high hyperglycemia in mothers and microsomia in the offspring..$^{19,20}$ Both macrosomic and microsomic infants may have long-term consequences at childhood, adolescence, and adulthood.

Our results showed that the body weight of the offspring of diabetic dams is lower than that observed in offspring of control dams as previously reported by Piazza et al. ${ }^{21}$.

Compelling evidence have shown that this maternal type 1 diabetes model can induce microsomia in the offspring, possibly as a result of intrauterine growth restriction with placental commitment, ${ }^{20,22,23}$ leading to long-term effects, especially in metabolic and neurologic parameters in the infants. ${ }^{24,25}$

The continuation of the hyperglycemic status in diabetic dams (D) after parturition seemed to contribute to the reduced growth during suckle, and pups remained small until weaning age (PND 21) as previously described by Chandna et al. ${ }^{26}$ The observed effects in offspring may not be only due to the gestational diabetes, but also to the malnutrition at early stages of offspring development, since that maternal type 1 diabetes can delay lactogenesis onset by affecting prolactin secretion and lactose, citrate, and total nitrogen concentrations in the milk. ${ }^{27}$

On the other hand, in the offspring of diabetic dams treated with ginger (D $+G$ ), our results showed an increase in body weight, which may be due to the decrease in maternal blood glucose after treatment with ginger extract and 
stimulation of lactation in these dams.

Developmental milestones play an important role in assessing the maturation of neonatal neurologic reflexes and serve as predictors of behavioral changes in adults. ${ }^{28}$

In our study, the offspring of diabetic dams presented neurodevelopmental delay in the negative geotaxis test according to and complementing the cognitive impairments reported by the clinic. ${ }^{29,24}$ Furthermore, a decrease in the object recognition index this is in agreement with the results of Kim et al. ${ }^{30}$ who have demonstrated, with the same experimental model, impaired memory in stepdown avoidance task on PND42.

The administration of ginger in diabetic dams during pregnancy had a beneficial effect on the neurological development of the pups, latency time in the negative geotaxis test was improved especially in females suggesting selective action on one sex. Also, there has been an increase in recognition memory in offspring of diabetic dams treated with ginger.

In addition, this plant extract and its active component, 6-gingerol, also inhibited cholinesterase activity, which increased acetylcholine, a neurotransmitter that plays an important role in learning and memory. ${ }^{31}$

There is considerable recent experimental evidence that ginger treatment has significantly improved word recognition, numerical alertness, choice reaction, digital working memory and spatial working memory scores in middle-aged women. ${ }^{32}$

These studies have shown that ginger can improve cognitive decline in the early stage of dementia in old age. Ginger intake during critical moments of fetal development is associated with an interaction with the neurophysiological processes of postnatal behavior.

Maternal diabetes is an intrauterine environment that is detrimental to the development of offspring, can cause brain damage and increases the risk of neurological disorders.

Our results indicate that severe maternal hyperglycemia during the fetal and perinatal period predisposes offspring to delayed physical development and neurodevelopmental disorders.

Our study clearly showed that treatment of diabetic pregnant rats with a ginger extract lowers blood glucose levels and can help prevent disorders in offspring. 


\section{REFERENCES}

1. Brahmachari, G. Chemistry and pharmacology of naturally occurring bioactive compounds, $1^{\text {st }}$ ed., Eds.; CRC Press: United states, 2013; pp 9-10.

2. Ekor, M. The growing use of herbal medicines: Issues relating to adverse reactions and challenges in monitoring safety, Front Pharmacol. 2014, 4, 177.

3. Shanmugam, K. R.; Mallikarjuna, K.; Kesireddy, N.; Sathyavelu.; Reddy, K. Neu-roprotective effect of ginger on anti-oxidant enzymes in streptozotocin-induceddiabetic rats, Food Chem Toxicol. 2011, 49, 893-897.

4. Huang, C. N.; Horng, J. S.; Yin, M. C. Antioxidative and antiglycative effects of sixorganosulfur compounds in low-density lipoprotein and plasma. J. Agric. Food Chem. 2004, 52, 3674-3678.

5. Young, H. V.; Luo, Y. L.; Cheng, H. Y.; Hsieh, W. C.; Liao, J. C.; Peng, W. C. Analgesic and anti-inflammatory activities of [6]-gingerol. J. Ethnopharmacol. 2oo5, 96, 207-210.

6. Veeresham, C. Natural Products Derived from Plants as a Source of Drugs. J. Adv. Pharm. Technol. Res. 2012, 3, 200-201.

7. Hiramatsu, Y.; Sekiguchi, N.; Hayashi, M.; Isshiki, K.; Yokota, G.L. Diacylglycerol production and protein Kinase $\mathrm{C}$ activity are increased in a model of diabetic embryopathy. Diabetes. 2002, 51, 2804-2810.

8. Freeman, M. E. The neuroendocrine control of the ovarian cycle of the rat. In: Knobi, E, Neil, J.D, (Eds.), The physiology of reproduction, 2nd edition, New York: Raven Press. 1994, 441-484.

9. Grozier, W. J. ; Pincus, G. La conduite géotropique des jeunes rats. J. Gen. Physiol. 1926, $10,257-269$.

10. Ennaceur, A.; Neave, N.; Aggleton, J. P. Spontaneous object recognition and object location memory in rats: the effets of lesions in the cingulated cotices, the medial prefrontal cortex, the cingulum bundle and the fornix. Exp. Brain Res. 1997, 113, 509-519.

11. Schulz, K. M.; Pearson, J. N.; Neeley, E. W.; Berger, R.; Leonard, S.; Adams, C. E.; Stevens, K. E. Maternal stress during pregnanacy causes sex-specific alterations in offspring memory performance, social interactions, indices of anxiety, and body mass. Physiol. Behav. 2011, 104, 340-.347.

12. Daisy, P.; Feril, G.; Jeeva, K. Hypolipidimic and hepatoprotective effects of cassia auriculatalinnbarck extracts on streptozotocin induced diabetics in male wistar albino rats. Asian $J$. Pharm. Clin. Res. 2013, 6, 43-48.

13. Frode, T. S.; Medeiros, Y. S. Animal models o test drugs with potential antidiabetic activity. J. Ethnopharmacol. 2008, 115, 173-183.

14. Szkudelsk, T. The mechanism of alloxan and streptozotocin action in B cells of the rat pancreas. Physiol. Res. 2001, 50, 537-546.

15. Davidoff, A. J.; Davidson, M. B.; Carmody, M. W.; David, M. E.; Ren, J. Diabetic cardiomyocyte dysfunction and myocyte insulin resistance: role of glucose-induced PKC activity. Mol. Cell. Biochem. 2004, 262, 155-163.

16. Rasmussen, P. Ginger-Zingiber officinale Roscoe, Zingiberaceae. J. Primary Health Care. 2011, 3, 235-236.

17. Riazur, R.; Akram, M.; Akhtar, N.; Jabeen, Q.; Saeed, T.; Ali Shah S. M. Zingiber officinale Roscoe (pharmacological activity). J. Med. Plants Res. 2011, 5, 344- 348. 
18. Marles, R. J.; Farnsworth, N. R. Antidiabetic plants and their active constituents. Phytomedicine. 1995, 2, 137-189.

19. Ornoy, A. Prenatal origin of obesity and their complications: gestational diabetes, maternal overweight and the paradoxical effects of fetal growth restriction and macrosomia. Reprod. Toxicol. 2011, 32, 205-212.

20. Rudge, M. V.; Piculo, F.; Marini, G.; Damasceno, D. C.; Calderon, I. M.; Barbosa, A. P. Translational research in gestational diabetes mellitus and mild gestational hyperglycemia: current knowledge and our experience. Arq. Bras. Endocrinol. Metabol. 2013, 57, 497-508.

21. Piazza, F. V.; Segabinazi, E.; Ferreira de Meireles, A. L.; Mega, F.; Spindler, C. F.; Augustin, O. A. Severe uncontrolled Maternal Hyperglycemia Induces Microsomia and Neurodevelopment Delay Accompanied by Apoptosis, Cellular Survival, and Neuroinflammatory Deregulation in Rat Offspring Hippocampus. Cell. Mol. Neurobiol. 2019, 39, 401-414.

22. Van Assche, F. A.; Holemans, K.; Aerts, L. Long-term consequences for offspring of diabetes during pregnancy. Br. Med. Bull. 2001, 6o, 173-182.

23. Volpato, G. T.; Damasceno, D. C.; Sinzato, Y. K.; Ribeiro, V. M.; Rudge, M. V.; Calderon, I. M. Oxidative stress status and placental implications in diabetic rats undergoing swimming exercise after embryonic implantation. Reprod. Sci. 2015, 22, 602-608.

24. Perna, R.; Loughan, A. R.; Le, J.; Tyson, K. Gestational diabetes: long-term central nervous system developmental and cognitive sequelae. Applied Neuropsychol. Child. 2015, 4, 217-220.

25. Hami, J.; Shojae, F.; Vafaee-Nezhad, S.; Lotfi, N.; Kheradmand, H.; Haghir, H. Some of the experimental and clinical aspects of the effects of the maternal diabetes on developing hippocampus. World J. Diabetes. 2015, 6, 412-422.

26. Chandna, A. R.; Kuhlmann, N.; Bryce, C. A.; Greba, Q.; Campanucci, V. A.; Howland, J. Chronic maternal hyperglycemia induced during mid-pregnancy in rats increases rage expression, augments hippocampal excitability, and alters behavior of the offspring. J. Neurosci. 2015, 303, 241-260.

27. Hartmann, P.; Cregan, M. Lactogenesis and the effects of insulin-dependent diabetes mellitus and prematurity. J. Nutr. 2001, 131, 3016-3020.

28. Stigger, F.; Lovatel, G.; Marques, M.; Bertoldi, K.; Moysés, F.; Elsner, V.; Siqueira, I. R.; Achaval, M.; Marcuzzo, S. Inflammatory response and oxidative stress in developing rat brain and its consequences on motor behavior following maternal administration of LPS and perinatal anoxia. Int. J. Dev. Neurosci. 2013, 31, 820-827.

29. DeBoer, T.; Wewerka, S.; Bauer, P. J.; Georgieff, M. K.; Nelson, C. A. Explicit memory performance in infants of diabetic mothers at 1 year of age. Dev. Med. Child Neurol. 2oo5, $47,525-531$.

30. Kim, Y. H.; Sung, Y. H.; Lee, H. H.; Ko, I. G.; Kim, S. E.; Shin, M. S.; Kim, B. K. Postnatal treadmill exercise alleviates short-term memory impairment by enhancing cell proliferation and suppressing apoptosis in the hippocampus of rat pups born to diabetic rats. J. Exerc. Rehabil. 2014, 10, 209-217.

31. Ghayur, M. N.; Gilani, A. H.; Ahmed, T. Muscarinic, $\mathrm{Ca}(++)$ antagonist and specific butyrylcholinesterase inhibitory activity of dried Ginger extract might explain its use in dementia. J. Pharm. Pharmacol. 20o8, 6o, 1375-1383.

32. Saenghong, N.; Wattanathorn, J.; Muchimapura, S.; Tongun, T.; Piyavhatkul, N.; Banchonglikitkul, C. Zingiber officinale improves cognitive function of the middle-aged healthy women. Evid. Based Complement. Alternat. Med. 2012, 2012, 1-9. 